\title{
OPTIMAL CONTROL FOR SYSTEMS GOVERNED BY DISCONTINUOUS NONLINEARITY
}

\author{
NGUYEN BUONG
}

\begin{abstract}
The aim of this paper is to present an existence theorem of optimal control for systems descrided by the operator equation of Hammerstein type $x+K F(u, x)=0$ with the discontinuous monotone nonlinear operator $F$ in $x$. Then, the theoretical result is applied to investigate an optimal control problem for system, where the state is written in the form of nonlinear integral equations in $L_{p}(\Omega)$.
\end{abstract}

\section{Introduction}

More and more technical and physical problems have been recently formulated in the form of equations of Hammerstein type, see, e.g., [2]-[4], [6]-[8], [10]-[18], [21], [22]. We often need to control such systems optimally. For the case when the nonlinear operator $F$ is smooth, such control problems are considered in [1], [9], [14] and [23]. From the optimal control point of view, the main difficulty consists of the fact that the nonlinear operator $F$ is generally discontinuous in $x$. This situation usually arises in optimal control for system with variable structure (see [11]). In this paper, for the last case, by using the method of monotone operators we shall prove an existence theorem of optimal control and give one application of the result.

Let $X$ be a real Banach space and $X^{*}$ be its dual which are uniformly convex. For the sake of simplicity, the norms of $X$ and $X^{*}$ will be denoted by one symbol $\|\cdot\|$. We write $\left\langle x^{*}, x\right\rangle$ instead of $x^{*}(x)$ for $x^{*} \in X^{*}$ and $x \in X$. Let $F: \mathcal{U}_{a d} \times X \rightarrow X^{*}$ be a discontinuous operator in $x$, where $\mathcal{U}_{a d} \subset U, U$ is another reflexive Banach space of controls, and $\mathcal{U}_{a d}$ is a convex, closed and nonempty set. Let $K: X^{*} \rightarrow X$ be a bounded (i.e. image of any bounded subset is bounded), nonegative and linear operator.

Consider the problem of optimal control: find $u_{0} \in \mathcal{U}_{a d}$ such that

$$
\begin{aligned}
J\left(u_{0}\right) & =\min J(u), \quad u \in \mathcal{U}_{a d} \\
J(u) & =J(u, x(u)),
\end{aligned}
$$

where $J: U \rightarrow R^{1}$ is a cost function having the following properties:

Received October 14, 1998.

1991 Mathematics Subject Classification. 49J20.

Key words and phrases. Monotone operators, uniform convex Banach spaces. 
- $J(u, x)$ is l.s.c. (lower semicontinuous) weakly in $u$ and in $x$, i.e. $u_{n} \rightarrow u, x_{n} \rightarrow$ $x \Rightarrow \liminf J\left(u_{n}, x_{n}\right) \geq J(u, x)$, and

- $J(u, x)$ is coercive in $u \in \mathcal{U}_{a d}$, i.e. $\liminf J(u, x)=+\infty$ as $\|u\| \rightarrow+\infty, u \in \mathcal{U}_{\text {ad }}$ uniformly with respect to $x$,

the symbols $\rightarrow$ and $\rightarrow$ denote convergence in norm and weak convergence, respectively, and the state of the considered system $x(u)$ is described by the operator equation

$$
x+K F(u, x)=0
$$

where $F(u, x)$ possesses the following properties:

i) $F: \mathcal{U}_{a d} \times X \rightarrow X^{*}$ is bounded,

ii) for every fixed $x \in X, F(\cdot, x)$ is weakly continuous in $u$, and

iii) for every fixed $u \in \mathcal{U}_{a d}, F(u, \cdot)$ is monotone in $x$, i.e.

$$
\langle F(u, x)-F(u, y), x-y\rangle \geq 0, \quad \forall_{u} \in \mathcal{U}_{a d}, \quad x, y \in X .
$$

\section{Main Result}

Integral equations of Hammerstein type with the nonlinear discontinuous operator $F$ was investigated in [8], [12], [20] by introducing a new concept of solution. But, throughout this paper the word 'solution' is meant in the classical sense.

Definition 1 (see [19]). A point $x \in X$ is called a point of $h$-continuity of the operator $G: X \rightarrow X^{*}$ if

$$
\forall l \in X \lim _{t \rightarrow 0_{+}}\langle G(x+t l), l\rangle=\langle G(x), l\rangle .
$$

A point $x \in X$ is called a point of discontinuity, if $x$ does not satisfy the condition in Definition 1.

Definition 2. A discontinuous point $x$ of $G$ is called regular, if

$$
\exists l \in X: \lim _{t \rightarrow 0_{+}}\langle G(x+t l), l\rangle<0 .
$$

Theorem 2.1. Assume that $K: X^{*} \rightarrow X$ is a linear, bounded and nonegative operator, conditions $i$ ) - iii) hold, all the discontinuous points of $F$ are regular, and that there exists a positive constant $r$ such that

$$
\langle F(u, x), x\rangle>0, \quad \text { if } \quad\|x\|>r, \quad \forall u \in \mathcal{U}_{a d} .
$$

Then, $E q$. (1.2) has a solution $x(u)$, for each $u \in \mathcal{U}_{\text {ad }}$.

Proof. As in [6], consider the regularized equation

$$
x+B_{n} F(u, x)=0, \quad B_{n}=B+\alpha_{n} V,
$$


where $V$ is the standard dual mapping of $X^{*}$, i.e.,

$$
\left\langle V\left(x^{*}\right), x^{*}\right)=\left\|V\left(x^{*}\right)\right\|\left\|x^{*}\right\|=\left\|x^{*}\right\|^{2}, \quad \forall x^{*} \in X^{*},
$$

and $\alpha_{n}$ is the sequence of positive real numbers such that $\alpha_{n} \rightarrow 0$, as $n \rightarrow+\infty$. Then, $R\left(B_{n}\right)=X, B_{n}^{-1}(0)=0, B_{n}^{-1}$ is an one-to-one mapping, and $B_{n}^{-1}$ is continuous (see [5]). Therefore, all the discontinuous points of $F$ in $x$ are the discontinuous points of $\tilde{B}_{n}+F$, and inversely, all the discontinuous points of $\tilde{B}_{n}+F$ are the discontinuous points of $F$, where $\tilde{B}_{n}(x)=-B_{n}^{-1}(-x)$. Obviously, we can rewrite Eq. (2.1) in the form

$$
\tilde{B}_{n}(x)+F(u, x)=0 .
$$

By virtue of [19], Eq. (2.2) has a unique solution, henthforth denoted by $x_{n}(u)$. Moreover, $\left\|x_{n}(u)\right\| \leq r, \forall n$. As $F$ is bounded, the sequence $\left\{F\left(u, x_{n}\right)\right\}$ is bounded, too. Without loss of generality, assume that

$$
x_{n} \rightarrow x_{0}, \text { and } F\left(u, x_{n}\right) \rightarrow y_{0}^{*} .
$$

From (2.1) it implies that

$$
x_{0}+B y_{0}^{*}=0 \text {. }
$$

Now, we have to prove that $y_{0}^{*}=F\left(u, x_{0}\right)$. Since $F$ is monotone, then

$$
\left\langle F(u, x)-F\left(u, x_{n}\right), x-x_{n}\right\rangle \geq 0, \quad \forall x \in X
$$

Hence,

$$
\left\langle F(u, x), x-x_{n}\right\rangle-\left\langle F\left(u, x_{n}\right), x\right\rangle \geq\left\langle F\left(u, x_{n}\right), B F\left(u, x_{n}\right)\right\rangle+\alpha_{n}\left\langle F\left(u, x_{n}\right), V F\left(u, x_{n}\right)\right\rangle .
$$

By passing $n \rightarrow+\infty$ in the last inequality, because of

$$
\begin{aligned}
& \liminf \left\langle F\left(u, x_{n}\right), B F\left(u, x_{n}\right)\right\rangle \geq\left\langle y_{0}^{*}, B y_{0}^{*}\right\rangle, \\
& \lim _{n \rightarrow+\infty} \alpha_{n}\left\langle F\left(u, x_{n}\right), V F\left(u, x_{n}\right)\right\rangle=0,
\end{aligned}
$$

and (2.3) we have

$$
\left\langle F(u, x)-y_{0}^{*}, x-x_{0}\right\rangle \geq 0 .
$$

Replacing, for any $l \in X$ and $t>0, x$ by $x_{0}+t l$ in (2.4) we see that

$$
\left\langle F\left(u, x_{0}+t l\right)-y_{0}^{*}, l\right\rangle \geq 0, \quad \forall l \in X .
$$

Hence, $x_{0}$ is the point of h-continuity of $F$. Consequently, from (2.4) and Minty's lemma (see [22]) $y_{0}^{*}=F\left(u, x_{0}\right)$. Theorem is proved.

Remark. If one of the operators $K$ and $F(u, \cdot)$ is strictly monotone, then the solution $x(u)$ is unique. 
Theorem 2.2. Assume that all the conditions in Theorem 2.1 hold, and that $J$ is l.s.c. weakly in $u, x$ and coercive in $u$, then Problem (1.1) has an optimal control $u_{0}$.

Proof. Let $u_{n}$ be a minimizing sequence of the functional $J(u)$. As $J(u)$ is coercive, the sequence $\left\{u_{n}\right\}$ is bounded. We denote by $x_{n}$ the solution of Eq. (2.1) with $u=u_{n}$. We shall prove that the sequence $\left\{x_{n}\right\}$ also is bounded. To do this, we construct the Banach space $Z=X \times X^{*}$ with the norm $\|z\|^{2}=\|x\|^{2}+\left\|x^{*}\right\|^{2}, z=\left[x, x^{*}\right], x \in X$ and $x^{*} \in X^{*}$. Consider the operator $\mathcal{A}(u, z):=\left[F(u, x), K x^{*}\right]+\left[-x^{*}, x\right]$. Obviously, $z_{n}=\left[x_{n}, x_{n}^{*}\right]$ is a solution of the equation

$$
\mathcal{A}\left(u_{n}, z_{n}\right)=0
$$

if and only if $x_{n}$ is a solution of the equation (2.1) with $u=u_{n}$. The space $Z$ has the dual space $Z^{*}=X^{*} \times X$ and the operator $\mathcal{A}: U \times Z \rightarrow Z^{*}$ is also a monotone operator in $z$ and weakly continuous in $u$ (see [6]). Certainly, $\mathcal{A}$ is discontinuous in $z$ because of discontinuity of $F$ in $x$.

On the other hand; from condition ii) we obtain

$$
\left(\gamma\left(\left\|x_{n}\right\|\right)-\left\|F\left(u_{n}, 0\right)\right\|\right)\left\|x_{n}\right\| \leq\left\langle\mathcal{A}\left(u_{n}, z_{n}\right)-\mathcal{A}\left(u_{n}, 0\right), z_{n}\right\rangle \leq\left\|F\left(u_{n}, 0\right)\right\|\left\|x_{n}\right\| .
$$

Therefore, the sequence $\left\{x_{n}\right\}$ also is bounded. Let $u_{n} \rightarrow u_{0}, z_{n} \rightarrow z_{0}:=\left[x_{0}, x_{0}^{*}\right]$. We shall prove that $x_{0}$ is a solution of (1.2) with $u=u_{0}$. Since $\left\langle\mathcal{A}\left(u_{n}, z_{n}\right), z-z_{n}\right\rangle=0$, then from

$$
\left\langle\mathcal{A}\left(u_{n}, z\right)-\mathcal{A}\left(u_{n}, z_{n}\right), z-z_{n}\right\rangle \geq 0, \quad \forall z \in Z
$$

it follows

$$
\left\langle\mathcal{A}\left(u_{0}, z\right), z-z_{0}\right\rangle \geq 0, \quad \forall z \in Z .
$$

Thus, $z_{0}$ can only be the point of h-continuity of the operator $\mathcal{A}$. So, $\mathcal{A}\left(u_{0}, z_{0}\right)=0$. In other words, $x_{0}=x\left(u_{0}\right)$ is a solution of (1.2) with $u=u_{0}$. From the weak 1.s.c. of $J$ and $x_{n} \rightarrow x_{0}$ (see [7]) we have

$$
J\left(u_{0}\right) \leq \liminf J\left(u_{n}\right) .
$$

Theorem is proved.

\section{Example}

Consider a system described by the nonlinear integral equation

$$
x(s)+\int_{\Omega} k(s, t) f(u, x(t)) d t=0,
$$

where the kernel function $k(s, t)$ is such that the operator $K$ defined by

$$
(K x)(s)=\int_{\Omega} k(s, t) x(t) d t
$$

is nonnegative, and $K$ acts from $L_{q}(\Omega)$ into $L_{p}(\Omega), L_{p}(\Omega)$ denotes the space of $p$ summable functions in the $\sigma$-finite measure set $\Omega \subset R^{n}$, with $p^{-1}+q^{-1}=1$, and the nonlinear function $f(u, t)$ satisfies the following conditions: 
a) $f(u, t) t \geq a_{0}|\hat{t}|^{p}+b_{0}|t|^{\gamma}+c_{0}, a_{0}>0, b_{0}<0, c_{0}<0, \gamma<p$,

b) $f(u, t)$ is not decreasing, and is right continuous in $t$, at the point of discontinuity $t_{0}$ $f\left(u, t_{0}-0\right) f\left(u, t_{0}\right)>0$, for every fixed $u \in \mathcal{U}_{a d}$,

c) $|f(u, t)| \leq a_{1}+b_{1}|t|^{p-1}, \forall t \in R^{1}, a_{1}+b_{1}>0, a_{1} \geq 0, b_{1} \geq 0$, and

d) $f(\cdot, x(t)): U \rightarrow L_{q}(\Omega)$ is, for every fixed $x(t) \in L_{p}(\Omega)$, compact.

Because of c) we define the operator $F: X=\mathcal{U}_{a d} \times L_{p}(\Omega) \rightarrow X^{*}=L_{q}(\Omega)$ as follows

$$
F(u, x)(t)=f(u, x(t)), \quad \forall x(t) \in L_{p}(\Omega),
$$

where $\mathcal{U}_{\text {ad }}$ is a convex, closed subset in a real reflexive Banach space of controls $U$. Then, Eq. (3.1) can be rewritten in the form (1.1), where the defined operator $F(u, x)$ possesses all the properties from Section 1. Indeed, condition a) guarantees the existence of $r$ in Theorem 2.1, the monotone property and the regularity of discontinuity points of $F(u, x)$ in $x$ follow from condition b) (see [19]), and remaining properties are verified on base of conditions c) and d). Therefore, for each fixed $u \in \mathcal{U}_{a d}$, Eq. (3.1) has only a solution, and the solution is unique if one of $K$ and $F$ is strictly monotone.

Moreover, we are given these additional data: $W$ is a real Banach space (observation space ), $C: L_{p}(\Omega) \rightarrow W$ a linear continuous operator, $w_{d} \in W$ a fixed element. Then, it is easy to see that the cost function

$$
J(u)=J(u, x)=\left\|C x-w_{d}\right\|_{W}^{\mu}+A\|u\|_{U}^{\theta},
$$

where $A, \mu, \theta$ are the positive constants, and $\|\cdot\|_{W}$ denotes the norm of $W$, is coercive in $u$ uniformly with respect to $x$ and l.s.c. weakly in $x$ and in $u$. Therefore, Probl.(3.1) and (3.2) has an optimal control $u_{0}$.

\section{References}

[1] D. E. Akbarov, Necessary condition of optimality in form of variational inequalities for objects described by equations of Hammerstein type, Cybern. and System Analysis, $\mathbb{1}(1996)$, 146-152(in Russian).

[2] H. Amann, Ein Existenz und Eindeutigkeitssatz fur die Hammersteinshe Gleichung in Banachraumen, Math. Z., $111(1969)$, 175-190.

[3] H. Amann, Uber die naherungsweise Losung nichlinearer Integralgleichungen, Numer. Math., 19 (1972), 29-45.

[4] H. Brezis and F. Browder, Nonlinear integral equations and systems of Hammerstein's type, Adv. Math., 10 (1975), 115-144.

[5] H. Brezis and M. Sibony, Méthod d'Approximation et d'Itération pour les opérateurs monotones, Arch. Rat. Mech. Anal., 28(1968), 59-82.

[6] N. Buong, On approximate solutions of Hammerstein equation in Banach spaces, Ukrainian Math. J., 8(1985), 1256-1260(in Russian).

[7] N. Buong, On solutions of the equations of Hammerstein type in Banach spaces, J. of Math. Computation \& Math. Physics, 25(1985), 1256-1280(in Russian).

[8] N. Buong, On solution of the operator equation of Hammerstein type with semimonotone and discontinuous nonlinearity, J. of Math., 12(1984), 25-28(in Vietnamese). 
[9] N. Buong, Concerning optimal control of one class of nonlinear equation in the Banach space, Ukrainian Math. J., 42(1990), 539-541(in Russian).

[10] N. Buong, On approximate solution for operator equation of Hammerstein type, J. of Comp. and Applied Math., 75(1996), 77-86.

[11] C. V. Emellianov, Systems of automatic control with variable structure, M. Nauka, 1967(in Russian).

[12] D. P. Gaidarov and P. K. Raguimkhanov, On integral inclusion of Hammerstein, Sibirian Math. J., 21(1980), 19-24(in Russian).

[13] C. Gupta, On nonlinear integral equations of Hammerstein type, Functional Analysis, Lecture Notes in Math. Springer-Verlage, New-York, 384(1973), 184-238.

[14] M. Joshi, On the existence of optimal control in Banach space, Bull. Austr. Math. Soc., $27(1983), 395-401$.

[15] H. Kaneko and Y. Xu, Degenerate kernel method for Hammerstein equation, Math. Comp., 56(1991), 141-148.

[16] H. Kaneko, R. Noren and Y. Xu, Numerical solutions for weakly singular Hammerstein equations and their supperconvergence, J. Int. Eqs. Appl., 4(1992), 391-407.

[17] S. Kumar, Superconvergence of a collocation-type method for Hammerstein equations, IMA Journal of Numerical Analysis, 7(1987), 313-325.

[18] C. D. Panchal, Existence theorems for equations of Hammerstein type, The Quart. J. of Math., 35(1984), 311-320.

[19] V. N. Pavlenko, Nonlinear equations with discontinuous operators in Banach spaces, Ukrainian Math. J., 31(1979), 569-572(in Russian).

[20] P. K. Raguimkhanov, Concerning an existence problem for solution of Hammerstein equation with discontinuous mappings, Izvestia Vuzov, Math., 10(1975), 62-70(in Russian).

[21] D. Vaclav, Monotone Operators and Applications in Control and Network Theory, Elsevier, New York, 1979.

[22] M. M. Vainberg, Variational Method and Method of Monotone Operators, Moscow, Nauka, 1972, (in Russian).

[23] L. V. Wolfersdorf, Optimal control of a class of process described by general integral equations of Hammerstein type, Math. Nachr., 71(1976), 115-141.

National Centre for Natural Sciences and Technology of Vietnam, Institute of Information Technology, Nghia do, Tu liem, Ha noi, Vietnam 10000.

Fax: (84)4-345217

e-mail: nbuong@math-ioit.ac.vn 